\title{
Turbulence mitigation in maximum-J stellarators with electron-density gradient
}

\author{
J.H.E. Proll ${ }^{\circledR 1}{ }^{\dagger}$, G.G. Plunk ${ }^{\circledR 2}$, B.J. Faber ${ }^{\circledR 3}$, T. Görler ${ }^{\circledR 4}$, P. Helander ${ }^{\circledR 2}$, \\ I.J. McKinney ${ }^{\circledR 3}$, M.J. Pueschel ${ }^{1,5,6}$, H.M. Smith ${ }^{2}$ and P. Xanthopoulos ${ }^{\circledR 2}$ \\ ${ }^{1}$ Science and Technology of Nuclear Fusion, Department of Applied Physics, Eindhoven University of \\ Technology, 5600 MB Eindhoven, The Netherlands \\ ${ }^{2}$ Max-Planck-Institut für Plasmaphysik, Wendelsteinstraße 1, 17491 Greifswald, Germany \\ ${ }^{3}$ Department of Engineering Physics, University of Wisconsin-Madison, Madison, WI 53706, USA \\ ${ }^{4}$ Max-Planck-Institut für Plasmaphysik, Boltzmannstraße 2, 85748 Garching, Germany \\ ${ }^{5}$ Dutch Institute for Fundamental Energy Research, 5612 AJ Eindhoven, The Netherlands \\ ${ }^{6}$ Institute for Fusion Studies, The University of Texas at Austin, Austin, TX 78712, USA
}

(Received 24 August 2021; revised 17 January 2022; accepted 17 January 2022)

In fusion devices, the geometry of the confining magnetic field has a significant impact on the instabilities that drive turbulent heat loss. This is especially true of stellarators, where the density-gradient-driven branch of the 'trapped electron mode' (TEM) is predicted to be linearly stable if the magnetic field has the maximum-J property, as is very approximately the case in certain magnetic configurations of the Wendelstein 7-X experiment (W7-X). Here we show, using both analytical theory and simulations, that the benefits of the optimisation of W7-X also serve to mitigate ion-temperature-gradient (ITG) modes as long as an electron density gradient is present. We find that the effect indeed carries over to nonlinear numerical simulations, where W7-X has low TEM-driven transport, and reduced ITG turbulence in the presence of a density gradient, giving theoretical support for the existence of enhanced confinement regimes, in the presence of strong density gradients (e.g. hydrogen pellet or neutral beam injection).

Key words: fusion plasma, plasma confinement, plasma instabilities

\section{Introduction}

In magnetic confinement fusion devices, there are usually three processes limiting the energy confinement: radiation losses, neoclassical transport, which encompasses collisional diffusion - including the effect of particle drifts that arise due to gradients and curvature of the confining magnetic field - and turbulence. While tokamaks are never critically affected by collisional transport owing to their axisymmetry, stellarators historically suffered from poor confinement due to the lack of symmetry and the resulting high neoclassical transport losses at high temperature. Optimised stellarators

$†$ Email address for correspondence: j.h.e.proll@tue.nl 
using the concepts of quasi-symmetry (Nührenberg \& Zille 1988; Boozer 1995), like the quasi-helically symmetric experiment HSX (Anderson et al. 1995), or quasi-isodynamicity (Helander \& Nührenberg 2009; Nührenberg 2010), like the recent superconducting stellarator Wendelstein 7-X (W7-X) (Beidler et al. 1990; Klinger et al. 2013), are designed to overcome the problem of large neoclassical transport (Canik et al. 2007; Beidler et al. 2011), rendering turbulence the dominant transport channel. As in tokamaks, ion-temperature-gradient (ITG) modes and trapped-electron modes (TEMs) have been identified as the most transport-relevant amongst the electrostatic instabilities. Recent research has focussed on studying the effects of the magnetic geometry available to stellarators on the instability of the ITG mode and TEM. For quasi-isodynamic and other omnigeneous stellarators, the second adiabatic invariant $\mathrm{J}$ of fast-bouncing particles such as electrons is constant on flux surfaces with flux surface label $\psi$ (Helander 2014) and depends through the velocity $v$ on the total energy $E$ with $\partial \mathrm{J} / \partial E>0$ at high normalised plasma pressure:

$$
\mathrm{J}=\int m v_{\|} \mathrm{d} l=\mathrm{J}(\psi, E),
$$

where $v_{\|}$denotes the velocity parallel to the magnetic field. If now an instability with frequency below the bounce frequency, $\omega \tau_{b} \ll 1$, moves a particle outwards by $\Delta \psi$, the energy $\Delta E$ necessary for this follows from the conservation of J (Helander et al. 2012):

$$
\Delta \mathrm{J}=\frac{\partial \mathrm{J}}{\partial \psi} \Delta \psi+\frac{\partial \mathrm{J}}{\partial E} \Delta E=0 \rightarrow \Delta E=-\frac{\partial \mathrm{J} / \partial \psi}{\partial \mathrm{J} / \partial E} \Delta \psi .
$$

This means the movement is at the expense of the instability and thus is stabilising if $\partial \mathbf{J} / \partial \psi<0$, i.e. if $\mathbf{J}$ has its maximum at the magnetic axis (Rosenbluth 1968). Proll et al. (2012) and Helander, Proll \& Plunk (2013) showed analytically that stellarators with the maximum-J property, where all trapped electrons can be shown to experience bounce-averaged 'good' curvature, $\omega_{* e} \bar{\omega}_{d e}<0$ (where $\omega_{* e}$ is the electron drift wave frequency and $\bar{\omega}_{d e}$ is the bounce-averaged magnetic drift frequency of the electrons), should be largely stable to collisionless electron-driven TEM thanks to the stabilising property of the trapped electrons. While the theory states that this resilience should hold for perfectly quasi-isodynamic stellarators with the maximum-J property, linear simulations (Proll, Xanthopoulos \& Helander 2013; Alcusón et al. 2020) showed that W7-X, which is only approximately quasi-isodynamic, indeed benefits from reduced TEM growth rates, too. These results are however only linear and raise the question whether the enhanced stability actually results in less turbulent transport. Recent nonlinear analytical theory using the concept of available energy (Helander 2017, 2020) and preliminary simulations (Helander et al. 2015; Xanthopoulos et al. 2020) have hinted that this is indeed the case. The present paper investigates the question directly, demonstrating the fully nonlinear effect of trapped electron stabilisation on TEM turbulence, and also how the effect extends to ITG turbulence, by comparing simulation results obtained in the high-mirror configuration of W7-X, HSX and the DIII-D tokamak (Luxon 2002). We also provide analytical arguments for why, in quasi-isodynamic configurations, trapped electrons also have a stabilising property for ITG modes.

\section{ITG-stabilisation through trapped electrons}

To argue why trapped electrons in maximum-J configurations are also stabilising for ITG modes, we investigate the ITG in two different limits: near marginal stability and in the strongly driven limit. Near marginal stability, we can revisit the derivation from Proll 
et al. (2012) and Helander et al. (2013), where we defined the rate of gyrokinetic energy transfer from the electrostatic field to species $a$ as

$$
P_{a}=e_{a} \operatorname{Im}\left\{\left(\mathrm{i} v_{\|} \nabla_{\|} g_{a}-\omega_{d a} g_{a}\right) \phi^{*} \mathrm{~J}_{0}\left(k_{\perp} v_{\perp} / \Omega_{a}\right)\right\} .
$$

Here, $e_{a}$ is the charge of particle species $a, \phi^{*}$ is the complex conjugate of the electrostatic potential, $\mathrm{J}_{0}$ is the zeroth-order Bessel function of the first kind, $v_{\perp}$ is the velocity perpendicular to the magnetic field, $\Omega_{a}$ is the Larmor frequency of species $a$ and $k_{\perp}$ is the magnitude of the perpendicular wave vector $\boldsymbol{k}_{\perp}=k_{\psi} \boldsymbol{\nabla} \psi+k_{\alpha} \nabla \alpha$ with $\alpha$ being the binormal coordinate used in the Clebsch representation of the magnetic field, $\boldsymbol{B}=$ $\nabla \psi \times \nabla \alpha$. The magnetic drift frequency is given by $\omega_{d a}=\boldsymbol{k}_{\perp} \cdot \boldsymbol{v}_{d a}$, with the drift velocity $\boldsymbol{v}_{d a}=\hat{\boldsymbol{b}} / \Omega_{a} \times\left(\left(v_{\perp}^{2} / 2\right) \nabla \ln B+v_{\|}^{2} \hat{\boldsymbol{b}} \cdot \nabla \hat{\boldsymbol{b}}\right)$, where $\hat{\boldsymbol{b}}$ is the unit vector along the magnetic field. Additionally, $g_{a}=f_{a 1}+\left(e_{a} \phi / T_{a}\right) f_{a 0}$ denotes the non-adiabatic part of the perturbed distribution function with the equilibrium distribution function $f_{a 0}$ being Maxwellian and $T_{a}$ the temperature of species $a$. We have also used the notation

$$
\{\cdots\}=\int_{-\infty}^{\infty} \frac{\mathrm{d} l}{B} \int(\cdots) \mathrm{d}^{3} v,
$$

i.e. we integrate over velocity space and along the field line in Ballooning space (Connor, Hastie \& Taylor 1979). It was shown that when using the general solutions of the linear electrostatic gyrokinetic equations as introduced by Connor, Hastie \& Taylor (1980) and Tang, Connor \& Hastie (1980), thus imposing no limit on the frequency $\omega$ of the instability, the energy transfer rate near marginal stability can be written as

$$
P_{a}=\omega \int_{0}^{\infty}\left(\omega-\omega_{* a}^{T}\right) \operatorname{Pos}_{a}(x, \omega) \mathrm{d} x,
$$

where $\operatorname{Pos}_{a}(x, \omega)$ is a positive definite function and the velocity-dependent diamagnetic frequency is given by $\omega_{* a}^{T}=\omega_{* a}\left[1+\eta_{a}\left(x^{2}-3 / 2\right)\right]$, with $\omega_{* a}=\left(T_{a} k_{\alpha} / e_{a}\right) \mathrm{d} \ln n_{a} / \mathrm{d} \psi$, $x^{2}=m_{a} v^{2} / 2 T_{a}$ and $\eta_{a}=\mathrm{d} \ln T_{a} / \mathrm{d} \ln n_{a}$ as the ratio between the temperature and density gradients (Proll et al. 2012; Helander et al. 2013). For a typical ITG mode travelling in the ion diamagnetic direction, i.e. $\omega \omega_{* i}>0$, and as long as $0 \leq \eta_{e}<2 / 3$, i.e. the electron temperature gradient is not too large compared with the density gradient, the energy transfer rate of the electrons will always be positive, $P_{e}>0$, meaning the electrons will draw energy from the mode rather than provide it. This finding does not only hold for maximum-J configurations but is, in fact, independent of any details of the geometry. This is perhaps a rather trivial finding when we only consider two species: to reach the point of marginal stability, where the sum of the energy transfer rates must be zero, only one species can provide the drive while the other will have to draw energy from the instability, and it is no surprise that the ions would be found to provide the drive for an ITG and the electrons to be stabilising. However, the result is also valid for more than two species, i.e. the stabilising property of the electrons remains even in situations with impurity species.

Far from marginality, we can still show that electrons are stabilising for ITGs in maximum-J configurations: as described by Plunk, Connor \& Helander (2017), in the strongly driven limit,

$$
\frac{\omega_{d a}}{\omega_{* a}} \ll 1
$$

which applies when gradients are large (and thus far from marginality), the dispersion relation of electrostatic modes can be cast into the form

$$
\omega=-\frac{1}{2 P}\left(Q \pm \sqrt{Q^{2}-4 P R}\right)
$$


with

$$
\begin{aligned}
P= & \int_{-\infty}^{\infty}\left[1+\frac{T_{e}}{T_{i}}\left(1-\Gamma_{0}\right)\right]|\phi|^{2} \frac{\mathrm{d} l}{B}-\frac{1}{2} \int_{1 / B_{\max }}^{\min } \sum_{j} \tau_{j}\left|\bar{\phi}_{j}\right|^{2} \mathrm{~d} \lambda \\
Q= & \frac{\omega_{* i} T_{e}}{T_{i}} \int_{-\infty}^{\infty}\left[\Gamma_{0}-\eta_{i} b\left(\Gamma_{0}-\Gamma_{1}\right)\right]|\phi|^{2} \frac{\mathrm{d} l}{B}+\frac{\omega_{* e}}{2} \int_{1 / B_{\max }}^{\min } \sum_{j} \tau_{j}\left|\bar{\phi}_{j}\right|^{2} \mathrm{~d} \lambda \\
R= & \frac{\omega_{* i} T_{e}}{T_{i}} \int_{-\infty}^{\infty} \frac{\omega_{d i}}{x^{2}(1-\lambda B)}\left[\Gamma_{0}-\frac{b\left(\Gamma_{0}-\Gamma_{1}\right)}{2}+\eta_{i} \Gamma_{0}(1-b)^{2}+\eta_{i} \Gamma_{1} b\left(\frac{3}{2}-b\right)\right]|\phi|^{2} \frac{\mathrm{d} l}{B} \\
& +\frac{3\left(1+\eta_{e}\right) \omega_{* e}}{4} \int_{1 / B_{\max }}^{\min } \sum_{j} \frac{\bar{\omega}_{d e j}}{x^{2}} \tau_{j}\left|\bar{\phi}_{j}\right|^{2} \mathrm{~d} \lambda
\end{aligned}
$$

where we have defined the bounce averages of the potential $\phi$ and the electron drift frequency $\omega_{d e}$ within a trapping well $j$ with magnetic field strength $B<1 / \lambda$ in the following form:

$$
\bar{\phi}_{j}(\lambda)=\int \frac{\phi(l) \mathrm{d} l}{\sqrt{1-\lambda B(l)}} / \int \frac{\mathrm{d} l}{\sqrt{1-\lambda B(l)}}=\int \frac{\phi(l) \mathrm{d} l}{\sqrt{1-\lambda B(l)}} / \tau_{j}
$$

where $\tau_{j}$ denotes the bounce time. For the integral over all trapped particles, we then need to sum over all possible trapping wells $j$ for a given pitch angle $\lambda$.

In the derivation of this dispersion relation, ion motion along the magnetic field has been neglected, which excludes slab ITG modes from the consideration but includes the curvature-driven ITG mode. While $P$ can be shown to be positive definite (Helander et al. 2013), $Q$ and $R$ can have either sign. Importantly, from (2.5), we see that we require $4 P R>$ $Q^{2}$ for $\omega$ having an imaginary part and thus an instability to exist. With $P$ positive, only a positive $R$ can lead to an instability, and a larger $R$ would mean a more unstable instability. As argued by Plunk et al. (2017), the electron contribution to $R$, i.e. the second term, is negative and thus stabilising for all $\eta_{e} \geq-1$, due to the proportionality to $\omega_{* e} \bar{\omega}_{d e j}$, which is negative for all particle orbits in maximum-J devices. Only the ion contribution, i.e. the first term in $R$, can be destabilising. Note that this form implies that stabilisation is expected from both the electron temperature and density gradients in the strongly driven limit.

These arguments thus predict enhanced stability of maximum-J configurations also to toroidal ITG modes, as soon as a density gradient is present, and thus add to the prediction of enhanced stability to TEMs shown analytically linearly (Proll et al. 2012; Helander et al. 2013) and nonlinearly (Helander 2017). The nonlinear argument in fact also extends to ITGs, as it can be shown that less energy (in the electrons) is available to drive instabilities when the density profile is slightly peaked than when it is flat (Helander 2020). Although they are not immune to ITG modes, maximum-J devices should thus benefit from somewhat reduced turbulence over a large range of parameter space, whenever a density gradient is present. In the absence of any electron drive, i.e. for flat density and electron temperature gradients, the presence of trapped electrons (second term in $P$ ) will deplete the Boltzmann electron density response. This will reduce $P$ and thus increase the growth rate of the ITG (see (2.5)), as will be seen when comparing ITG simulations with adiabatic and kinetic electrons. 


\section{Simulation set-up}

Collisionless electrostatic simulations were performed with the flux-tube version of the Eulerian code GENE (Jenko et al. 2000, see genecode.org for code details and access), which solves the gyrokinetic equation together with Maxwell's equations and incorporates realistic geometry when coupled to the GIST code (Xanthopoulos et al. 2009). For W7-X and HSX, the flux tubes studied cross the outboard midplane in the bean-shaped cross-section of the stellarator at the midpoint of the flux tube, as these flux tubes have been found to be the most unstable ones to ITG modes and TEMs in previous studies (Proll et al. 2013; Faber et al. 2015). While for W7-X, one poloidal turn has found to be sufficient for convergence, for HSX 4,poloidal turns have been found to be necessary (Faber et al. 2018). In all three devices, the flux surface chosen was at half-normalised toroidal flux, i.e. $s=\psi / \psi_{0}=0.5$. For more information about the geometry, the reader is referred to Proll et al. (2013) for DIII-D and W7-X and to Faber et al. (2018) for HSX. ITG turbulence was modelled with both adiabatic (ITG-ae) and kinetic (ITG-ke) electrons, and TEM with a pure density gradient; the resolution was chosen as seen in table 1 . There, $n z$ refers to the number of grid points along a field line, $n k x$ the number of grid points in the radial direction, $n k y$ to the number of Fourier modes in the binormal direction (i.e. perpendicular to both the field line and the radial direction), $n v$ to the number of grid points in the direction of parallel velocity $v_{\|}, n w$ to that in the direction of the magnetic moment $\mu$ and kymin to the minimum value of the wavenumber in units of inverse gyroradius.

For TEMs in HSX, $k_{y} \rho_{s}=0.1$ was found to be sufficient as the smallest binormal wavenumber. The simulations are performed with a realistic mass ratio for hydrogen plasmas of $m_{e} / m_{i}=1 / 1836$ and a temperature ratio of $T_{e} / T_{i}=1,{ }^{1}$ where $m_{e}$ and $m_{i}$ are the electron mass and the ion mass, respectively, and $T_{e}$ and $T_{i}$ the electron and ion temperatures, respectively. To assess the relative strength of the turbulence in different devices and for different types of drive, we will compare the sum of the electrostatic heat fluxes for both species $s, Q=\sum_{s} Q_{s}$, and the particle flux $\Gamma=\Gamma_{e}=\Gamma_{i}$, defined as follows:

$$
\begin{gathered}
\frac{\left\langle Q_{s}\right\rangle}{Q_{g b}}=\frac{\left\langle\int \mathrm{d}^{3} v \frac{1}{2} m_{s} v^{2} f_{s, 1} v_{d, r}\right\rangle}{Q_{g b}}, \\
\frac{\left\langle\Gamma_{s}\right\rangle}{\Gamma_{g b}}=\frac{\left\langle\int \mathrm{d}^{3} v f_{s, 1} v_{d, r}\right\rangle}{\Gamma_{g b}},
\end{gathered}
$$

with $f_{s, 1}$ as the fluctuating part of the particle distribution function of species $s, v_{d, r}$ is approximated by the radial component of the generalised $\boldsymbol{E} \times \boldsymbol{B}$ velocity, $m_{s}$ is the particle mass and where $\langle\cdots\rangle$ denotes the average over the flux tube. Both fluxes are normalised to gyro-Bohm units,

$$
\begin{aligned}
& Q_{g b}=n_{i} T_{i}^{5 / 2} m_{i}^{-3 / 2} /\left(\Omega_{i}^{2} a^{2}\right), \\
& \Gamma_{g b}=n_{i} T_{i}^{3 / 2} m_{i}^{-3 / 2} /\left(\Omega_{i}^{2} a^{2}\right),
\end{aligned}
$$

with ion density $n_{i}$, ion temperature $T_{i}$, ion mass $m_{i}$, minor radius $a$ and ion gyro frequency $\Omega_{i}$ typically evaluated at the magnetic axis. In this formulation, differences in surface-to-volume ratio are not accounted for. To compensate for that, factors of 5 or 4 for W7-X or HSX, respectively, would have to be used when comparing against DIII-D.

\footnotetext{
${ }^{1}$ In HSX experiments, the electron temperature $T_{e}$ usually exceeds the ion temperature $T_{i}$, but we always set them equal since our aim is to study the effect of magnetic geometry rather than modelling specific experiments.
} 
ITG-ae

DIII-D $\quad 192,64,64,48,20,0.05$

HSX $\quad 128,32,512,48,8,0.1$

W7-X $384,64,128,48,20,0.05$
ITG-ke

$192,64,64,48,20,0.05$

$128,48,512,48,8,0.1$

$256,64,96,48,10,0.05$ $\nabla n$ TEM

$192,48,64,48,20,0.05$

$128,32,256,32,8,0.1$

$256,64,96,48,10,0.05$

TABLE 1. Resolution for turbulence simulations ( $\mathrm{nkx}, \mathrm{nky}, \mathrm{nz}, \mathrm{nv}, \mathrm{nw}, \mathrm{kymin}$ ) for ITGs with adiabatic (ITG-ae) and kinetic (ITG-ke) electrons and TEM with a pure density gradient.

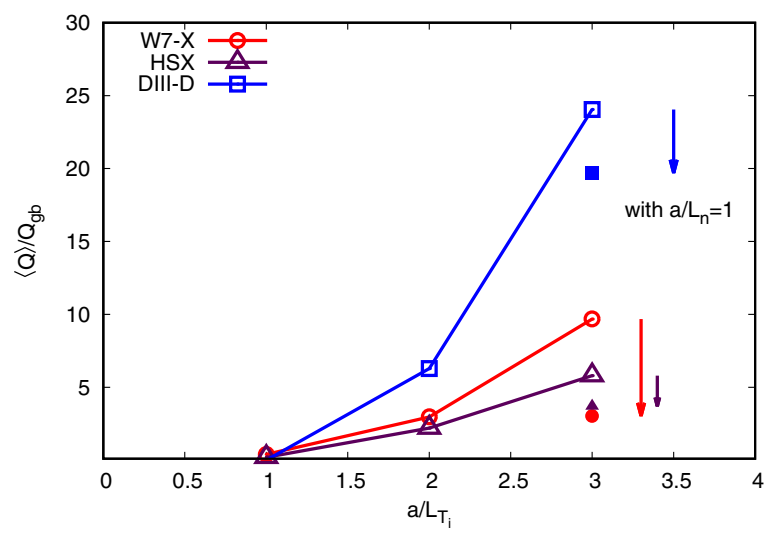

FIGURE 1. ITG adiabatic electrons: Flux-tube-averaged and time-averaged (in the quasi-stationary phase) normalised heat fluxes in W7-X, HSX and DIII-D for ion-temperature-gradient-driven turbulence with adiabatic electrons. The full symbols show how the heat fluxes change for an ion-temperature gradient of $a / L_{T_{i}}=3$ once a small density gradient $a / L_{n}=1$ is present.

For the linear simulations, we typically scan over the binormal wavenumber $k_{y} \rho_{s}$, where $\rho_{s}=\sqrt{T_{i} / m_{i}} / \Omega_{i}$, and we use the radial mode number $k_{x} \rho_{s}=0$.

\section{Nonlinear simulation results}

\subsection{Ion-temperature-gradient (ITG) modes}

First, we study the effect of the magnetic geometry on ITG turbulence by varying the ion temperature gradient $a / L_{T_{i}}=-a \mathrm{~d} \ln T_{i} / \mathrm{d} r$ while setting the electron-temperature gradient and the density gradient to zero. It is found that the heat flux of turbulence resulting from ITGs with adiabatic electrons (ITG-ae) is smaller in both stellarators than in DIII-D, see figure 1. The difference can likely be attributed to the strong local shear and low global shear in both stellarator devices (Plunk et al. 2014) - with HSX having particularly low global shear, also compare McKinney et al. (2019) - as well as generally smaller local curvature in W7-X. This difference is also reflected in the linear growth rates (for W7-X and DIII-D, see Proll et al. 2013). If we now add a density gradient $a / L_{n}=1$ for the case with $a / L_{T_{i}}=3$, we see the typical (Coppi, Rosenbluth \& Sagdeev 1967) partial stabilisation of ITG through the density gradient, resulting in reduced heat fluxes in all three devices.

Returning to the scenario with a flat density profile, the aforementioned inter-machine trend changes significantly if we also consider kinetic electrons: Initially we note that with kinetic electrons, the relative magnitude of the fluxes - now also the particle flux 
(a)

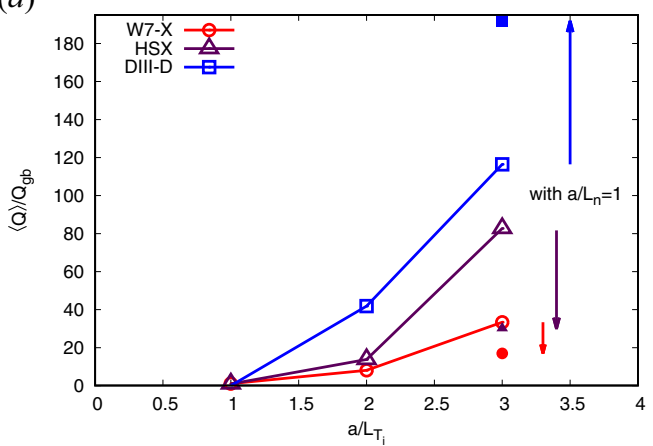

(b)

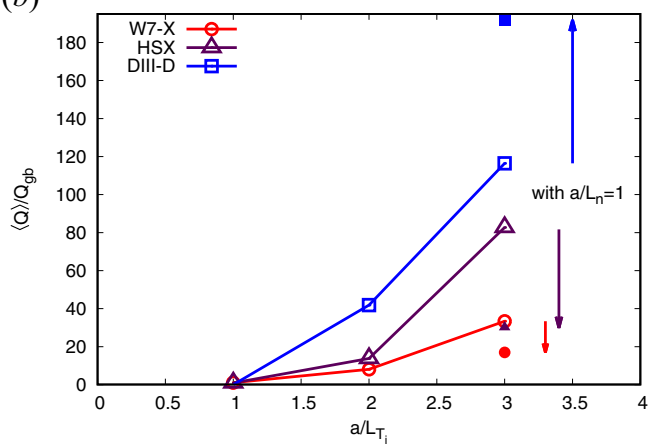

FIGURE 2. ITG kinetic electrons: flux-tube-averaged and time-averaged (in the quasi-stationary phase) normalised heat fluxes $(a)$ and particle fluxes $(b)$ in W7-X, HSX and DIII-D for ion-temperature-gradient-driven turbulence with kinetic electrons. The full symbols show how the fluxes change for an ion-temperature gradient of $a / L_{T_{i}}=3$ once a small density gradient $a / L_{n}=1$ is present.

in addition to the heat flux, see figure 2 - between the devices remains similar, with DIII-D having the highest fluxes and W7-X having the lowest (even though the particle fluxes of HSX are only barely larger than those of W7-X), and that all heat fluxes have increased compared with the case of adiabatic electrons, with the increase being strongest for HSX and weakest for W7-X. This increase in ITG activity when going from adiabatic to kinetic electrons is also observed in the linear growth rates and was discussed above. Now, if again a density gradient $a / L_{n}=1$ is added for the case with kinetic electrons and an ion-temperature gradient $a / L_{T_{i}}=3$, we observe that the three devices behave very differently: for DIII-D, both the heat flux and the particle flux increase strongly. This can be explained by trapped-electron-modes being destabilised (as is also seen in the linear growth rates, see figure 3), which then contribute to both fluxes. In W7-X and HSX, we see the opposite behaviour: the particle fluxes remain nearly constant, and the heat fluxes even decrease. This is rather remarkable, and to understand it, one needs to consider the linear growth rates for the ITGs with and without a density gradient, see figure 3. In W7-X, we see a reduction in linear growth rates once a density gradient is present, but not in DIII-D or in HSX. We attribute this to the high degree of maximum-J property of W7-X, which leads to a large fraction of trapped electrons having favourable curvature and thus being stabilising, as discussed above. An alternate explanation (Kotschenreuther et al. 2020) involves enhanced electron adiabaticity; assessing the relative effectiveness of these mechanisms is beyond the scope of the present work. As this reduction in growth rates is only seen at low wavenumbers $\left(k_{y} \rho_{s} \approx 0.1-0.2\right)$, the effect may be insufficient in fully explaining the reduction in nonlinear heat flux. However, to properly appreciate the linear effect of the maximum-J property, one needs to contrast the mild stabilisation of W7-X with the robust destabilisation for all wavenumbers in HSX, as expected when the TEM drive is increased, discussed immediately hereafter. Thus, one may conclude that the response of the heat flux of $\mathrm{W} 7-\mathrm{X}$ to increased density gradient indeed benefits from the stabilising influence of the maximum-J property.

In HSX, no linear stabilisation is observed but rather a destabilisation like in DIII-D, which is very well understandable as HSX is far from maximum-J. However, the nonlinear heat and particle fluxes show the opposite behaviour and decrease or remain constant, respectively, rather than increase, just like in W7-X. We thus note that in HSX, a strong 
(a)

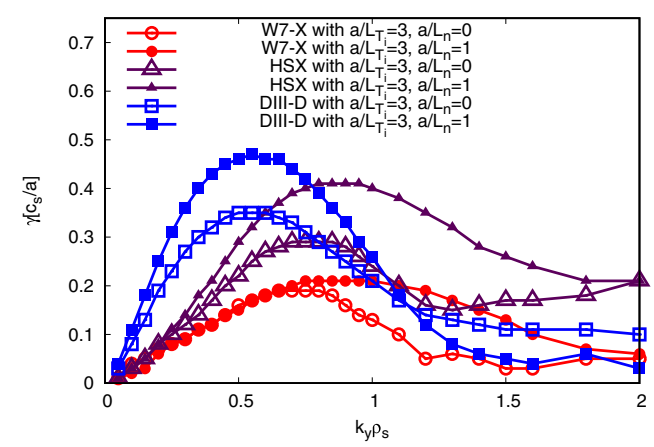

(b)

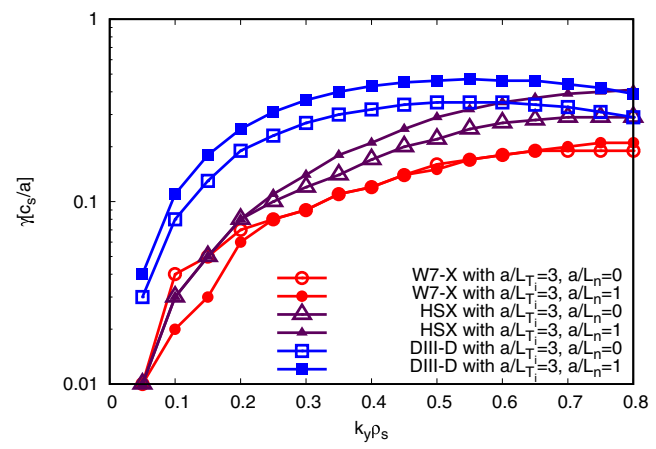

FIGURE 3. Normalised growth rates $\gamma$ of ITGs with kinetic electrons (ITG-ke) at $a / L_{T_{i}}=3$ with (full symbols) and without (open symbols) added density gradient of $a / L_{n}=1$ in W7-X, HSX and DIII-D, for a large range of wavenumbers $(a)$ and only the small wavenumbers $(b)$.

nonlinearly stabilising mechanism exists in the form of enhanced energy transfer to stable eigenmodes (Hegna, Terry \& Faber 2018; Terry et al. 2018), as measured, e.g. by the triplet correlation time (Terry et al. 2018; Whelan, Pueschel \& Terry 2018; Li et al. 2021; Pueschel, Li \& Terry 2021). The process underlying the substantial nonlinear stabilisation observed in HSX is likely also at work in W7-X, though to a smaller degree due to, among other factors, the differences in magnetic shear. The delicate task of evaluating the relative importance of the linear and nonlinear stabilisation will be left for future work. We note the possibility that both effects may be exploited in future stellarator designs.

\subsection{Trapped-electron modes (TEM)}

To study density-gradient-driven TEMs, both temperature gradients are set to zero and only the density gradient is varied. The heat and particle fluxes in both stellarators are up to two orders of magnitude smaller than that in the DIII-D geometry (see figure 4). In $\mathrm{W} 7-\mathrm{X}$, the explanation for the low fluxes lies again with the high degree of maximum-J property: not only are the linear growth rates of the density-gradient-driven TEM much smaller than in the two other devices (figure 5) - as predicted (Proll et al. 2012; Helander et al. 2013)- but we conclude that the enhanced stability also holds nonlinearly. One surprising observation is that in $\mathrm{W} 7-\mathrm{X}$, the fluxes at $a / L_{n}=1$ are slightly larger than those at $a / L_{n}=2$ (figure 4 ). This cannot be explained by the difference in linear growth rates (figure 6), which increase monotonically with increasing density gradient, as expected. We do, however, observe distinctly different modes at large perpendicular scales $\left(k_{y} \rho_{s} \leq 0.5\right.$ with $\left.k_{x} \rho_{s}=0\right)$ ): for both $a / L_{n}=2$ and $a / L_{n}=3$, the modes at these large scales propagate in the electron diamagnetic direction and have recently been classified as 'Universal instabilities' (Cheng \& Chen 1980; Chowdhury et al. 2010; Helander \& Plunk 2015; Landreman, Antonsen \& Dorland 2015a; Landreman, Plunk \& Dorland 2015b; Costello, Proll \& Plunk 2021), while the mode that is seen at smaller scales in all cases, but that also dominates the large scales at $a / L_{n}=1$, propagates in the ion diamagnetic direction and is most likely the ion-driven trapped electron mode (iTEM) described by Plunk et al. (2017). Both types of mode may have different nonlinear properties and therefore lead to differences in saturated amplitude. For HSX, understanding the low heat flux requires taking into account nonlinear saturation mechanisms. Just as for the ITG simulations with a density gradient present, the growth rates are much higher than in W7-X due to the lack of the maximum-J property. Even though the TEMs in HSX have shorter wavelengths 
(a)

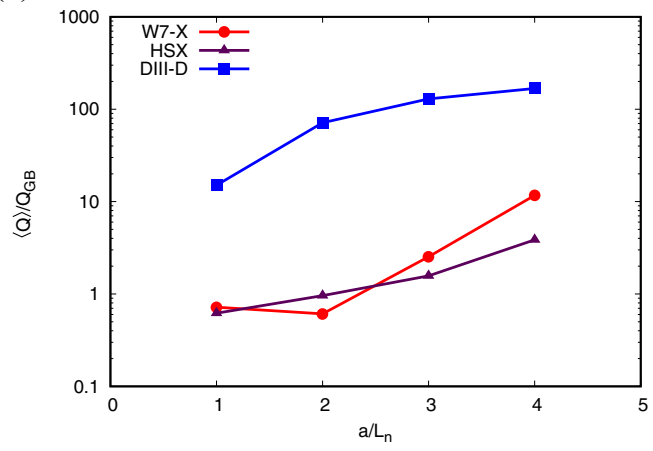

(b)

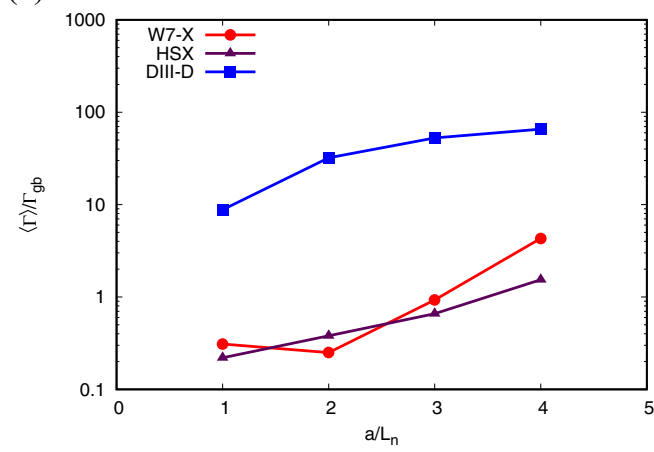

FIGURE 4. TEM: flux-tube-averaged and time-averaged (in the quasi-stationary phase) normalised heat fluxes $(a)$ and particle fluxes $(b)$ in W7-X, HSX and DIII-D for density-gradient-driven turbulence with kinetic electrons.
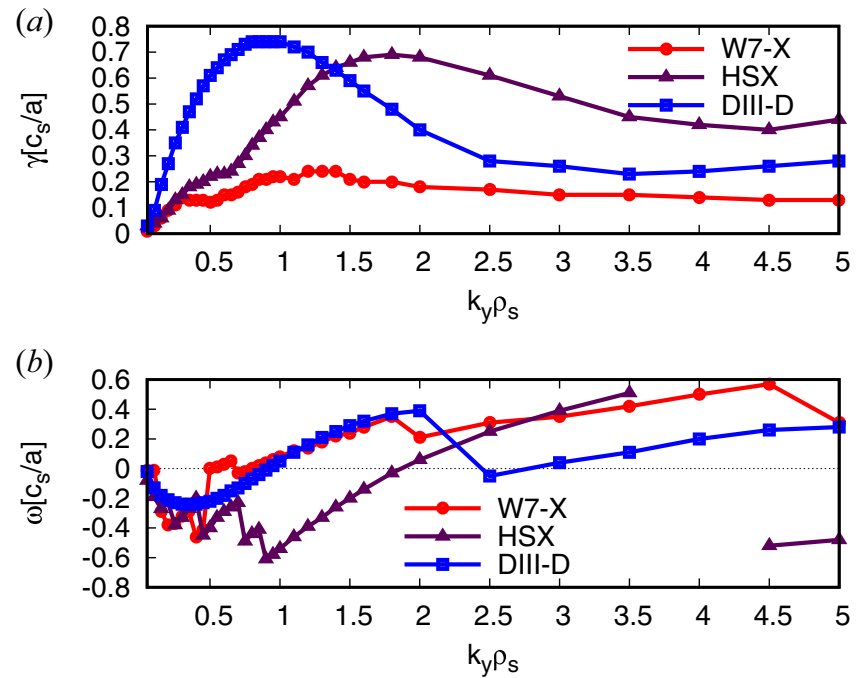

FIGURE 5. Normalised growth rates $\gamma$ and real frequencies $\omega$ of density-gradient-driven modes in W7-X, HSX and DIII-D for $a / L_{n}=3$.

than in DIII-D, a simple quasi-linear theory or mixing-length estimate cannot explain why the heat flux in HSX is comparable to, or even smaller than, that in W7-X. However, at very low background magnetic shear as in HSX, nonlinear energy transfer to stable eigenmodes can be enhanced, leading to lower-than-expected heat flux. Note that similar nonlinearly stabilising features have been seen in ITG turbulence comparisons between HSX and an axisymmetric configuration (McKinney et al. 2019). One final observation regarding $\mathrm{W} 7-\mathrm{X}$ is that, in spite of the TEM heat flux being small compared with the ITG heat flux or the heat fluxes in DIII-D, it is still large compared with the neoclassical flux as calculated by the SFINCS code (Landreman et al. 2014) at typical values of density $n=10^{19} \mathrm{~m}^{-3}$ and temperature $T=1-5 \mathrm{keV}$ (see figure 7 ). This clearly supports turbulent transport as the dominant transport channel in W7-X, and is very much in line with the experimental observations (Dinklage et al. 2018), where it was found that the heat flux calculated by neoclassical theory is not sufficient to explain experimental measurements. 

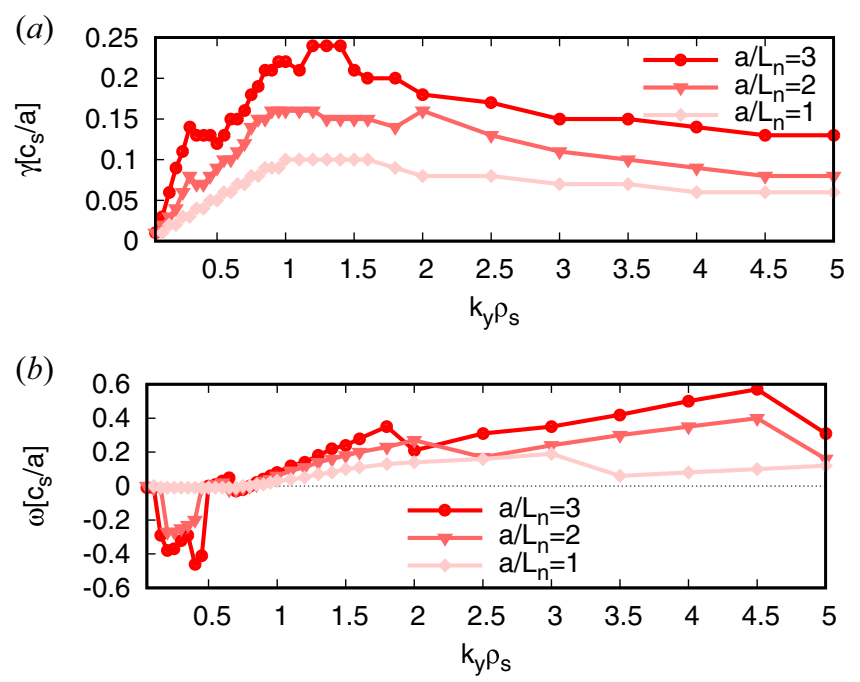

FIGURE 6. Normalised growth rates $\gamma$ and real frequencies $\omega$ for density gradients $a / L_{n}=1,2,3$ in W7-X.

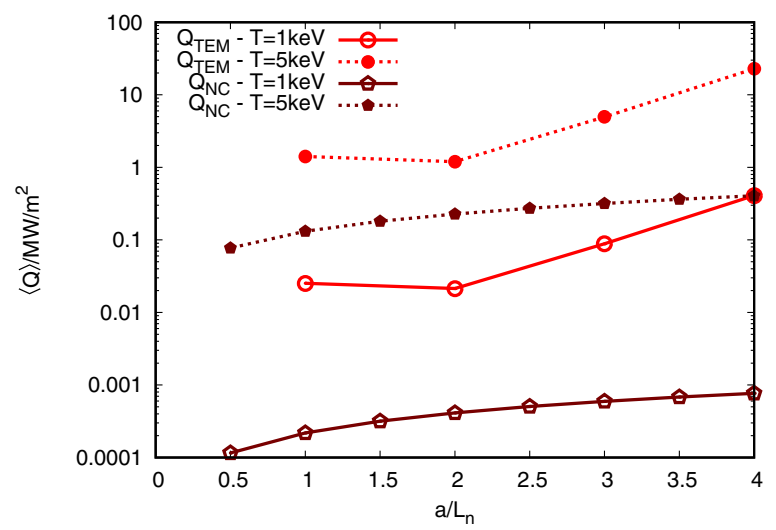

FIgURE 7. TEM turbulent heat flux $Q_{T E M}$ (red circles) and neoclassical heat flux $Q_{N C}$ (maroon pentagons), respectively, in W7-X for different density gradients. The usual normalisation in gyro-Bohm units has been transformed into MW m ${ }^{-2}$, assuming a density of $n=5 \times 10^{19} \mathrm{~m}^{-3}$ and temperatures of $T=1 \mathrm{keV}$ and $T=5 \mathrm{keV}$.

In summary, we find that in W7-X, the turbulent heat flux of both density-gradient-driven TEM and ITGs with a small density gradient is much lower than in a tokamak geometry. We attribute this enhanced stability to the maximum-J property of W7-X, though also enhanced electron adiabaticity (Kotschenreuther et al. 2020) cannot be ruled out as a possible explanation. HSX seems to benefit from a more powerful saturation mechanism, despite lacking maximum-J optimisation. This suggests that turbulence in stellarators like W7-X or HSX can be mitigated by increasing the density gradient, for example, through pellet injection, as recently reported for W7-X (Bozhenkov et al. 2020), thus providing a path toward enhanced fusion performance. 


\section{Acknowledgements}

The authors would like to thank the GENE team - most notably D. Told, F. Jenko, H. Doerk and A. Bañón-Navarro - for their efforts and support. We would also like to thank G. Hammett, S. Lazerson, H. Mynick, J. Citrin and M. Kotschenreuther for lots of stimulating and helpful discussions. The simulations were performed on MPCDFs Hydra and Helios at IFERC-CSC.

Editor William Dorland thanks the referees for their advice in evaluating this article.

\section{Funding}

This work has been carried out within the framework of the EUROfusion Consortium and has received funding from the Euratom research and training programme 2014-2018 and 2019-2020 under grant agreement No. 633053. The views and opinions expressed herein do not necessarily reflect those of the European Commission.

\section{Declaration of interests}

The authors report no conflict of interest.

\section{REFERENCES}

Alcusón, J.A., Xanthopoulos, P., Plunk, G.G., Helander, P., Wilms, F., Turkin, Y., Stechow, A.V. \& GRUlKe, O. 2020 Suppression of electrostatic micro-instabilities in maximum-J stellarators. Plasma Phys. Control. Fusion 62 (3), 035005.

Anderson, F.S.B., Almagri, A.F., Anderson, D.T., Matthews, P.G., Talmadge, J.N. \& Shohet, J.L. 1995 The helically symmetric experiment, (HSX) goals, design and status. Fusion Technol. 27 (3 T), 273-277.

Beidler, C.D., Grieger, G., Hernegger, F., Harmeyer, E., Kisslinger, J., Lotz, W., MaAßberg, H., Merkel, P., NÜhrenberg, J., Rau, F., et al. 1990 Physics and engineering design for Wendelstein VII-X. Fusion Technol. 17 (1), 148-168.

Beidler, C.D., Allmaier, K., Isaev, M.Yu., Kasilov, S.V., Kernbichler, W., Leitold, G.O., MaAßberg, H., Mikkelsen, D.R., Murakami, S., Schmidt, M., et al. 2011 Benchmarking of the mono-energetic transport coefficients - results from the international collaboration on neoclassical transport in stellarators (ICNTS). Nucl. Fusion 51 (7), 076001.

Boozer, A.H. 1995 Quasi-helical symmetry in stellarators. Plasma Phys. Control. Fusion 37 (11A), A103-A117.

Bozhenkov, S.A., Kazakov, Y., Ford, O.P., Beurskens, M.N.A., Alcusón, J.A., Alonso, J.A., BAldzuhn, J., Brandt, C., Brunner, K.J., DAmM, H., et al. 2020 High-performance plasmas after pellet injections in Wendelstein 7-X. Nucl. Fusion 60 (6).

Canik, J.M., Anderson, D.T., Anderson, F.S.B., Likin, K.M., Talmadge, J.N. \& Zhai, K. 2007 Experimental demonstration of improved neoclassical transport with quasihelical symmetry. Phys. Rev. Lett. 98 (February), 1-4.

Cheng, C.Z. \& Chen, L. 1980 Unstable universal drift eigenmodes in toroidal plasmas. Phys. Fluids 23 (9), 1770-1773.

Chowdhury, J., Ganesh, R., Brunner, S., Vaclavik, J. \& Villard, L. 2010 Toroidal universal drift instability: a global gyrokinetic study. Phys. Plasmas 17 (10), 102105.

CONNOR, J.W., HASTIE, R.J. \& TAYLOR, J.B. 1979 High mode number stability of an axisymmetric toroidal plasma. Proc. R. Soc. Lond. A 365 (1720), 1-17.

Connor, J.W., Hastie, R.J. \& TAYlor, J.B. 1980 Stability of general plasma equilibria. III. Plasma Phys. 22 (7), 757-769.

Coppi, B., Rosenbluth, M.N. \& Sagdeev, R.Z. 1967 Instabilities due to temperature gradients in complex magnetic field configurations. Phys. Fluids 10 (3), 582-587. 
Costello, P., Proll, J.H.E. \& Plunk, G.G. 2021 The universal instability in Wendelstein 7-X. (in preparation).

Dinklage, A., Beidler, C.D., Helander, P., Fuchert, G., Maaßberg, H., Rahbarnia, K., Sunn Pedersen, T., Turkin, Y., Wolf, R.C., Andreeva, T., et al. 2018 Magnetic configuration effects on the Wendelstein 7-X stellarator. Nat. Phys. 14 (8), 855-860.

Faber, B.J., Pueschel, M.J., Proll, J.H., Xanthopoulos, P., Terry, P.W., Hegna, C.C., Weir, G.M., LiKin, K.M. \& TAlmadge, J.N. 2015 Gyrokinetic studies of trapped electron mode turbulence in the Helically Symmetric eXperiment stellarator. Phys. Plasmas 22 (7), 072305.

Faber, B.J., Pueschel, M.J., Terry, P.W., Hegna, C.C. \& Roman, J.E. 2018 Stellarator microinstabilities and turbulence at low magnetic shear. J. Plasma Phys. 84 (5), 1-28.

Hegna, C.C., Terry, P.W. \& FABER, B.J. 2018 Theory of ITG turbulent saturation in stellarators: identifying mechanisms to reduce turbulent transport. Phys. Plasmas 25 (2), 022511.

Helander, P. 2014 Theory of plasma confinement in non-axisymmetric magnetic fields. Rep. Prog. Phys. 77, 087001 .

Helander, P. 2017 Available energy and ground states of collisionless plasmas. J. Plasma Phys. 83 (4), 1-20, arXiv:1706.05219.

Helander, P. 2020 Available energy of magnetically confined plasmas. J. Plasma Phys. 86 (2), 905860201, arXiv:2001.02494.

Helander, P., Beidler, C.D., Bird, T.M., Drevlak, M., Feng, Y., Hatzky, R., Jenko, F., Kleiber, R., Proll, J.H.E., Turkin, Y., et al. 2012 Stellarator and tokamak plasmas: a comparison. Plasma Phys. Control. Fusion 54 (12), 124009.

Helander, P., Bird, T., Jenko, F., Kleiber, R., Plunk, G.G., Proll, J.H., Riemann, J. \& Xanthopoulos, P. 2015 Advances in stellarator gyrokinetics. Nucl. Fusion 55 (5), 053030.

Helander, P. \& NÜHREnBerg, J. 2009 Bootstrap current and neoclassical transport in quasi-isodynamic stellarators. Plasma Phys. Control. Fusion 51 (5), 055004.

Helander, P. \& Plunk, G.G. 2015 The universal instability in general geometry. Phys. Plasmas 22 (9), 090706, arXiv: 1506.09098.

Helander, P., Proll, J.H.E. \& Plunk, G.G. 2013 Collisionless microinstabilities in stellarators. I. Analytical theory of trapped-particle modes. Phys. Plasmas 20 (12), 122505.

Jenko, F., Dorland, W., Kotschenreuther, M. \& Rogers, B.N. 2000 Electron temperature gradient driven turbulence. Phys. Plasmas 7 (5), 1904.

Klinger, T., Baylard, C., Beidler, C.D., Boscary, J., Bosch, H.S., Dinklage, A., Hartmann, D., Helander, P., MaßBerg, H., Peacock, A., et al. 2013 Towards assembly completion and preparation of experimental campaigns of Wendelstein $7-\mathrm{X}$ in the perspective of a path to a stellarator fusion power plant. Fusion Engng Des. 88 (6-8), 461-465.

Kotschenreuther, M., Liu, X., Hatch, D.R., Mahajan, S.M., Pueschel, M.J., Halfmoon, M., Zarnstorff, M., Garofalo, A., McClenaghan, J., McKinney, I.J., Qian, J., et al. 2020 Regimes of weak itg/tem for transport barriers without velocity shear. In APS-DPP conference, VP14.00017.

LANDREMAN, M., ANTONSEN, T.M. \& DoRlAND, W. 2015a Universal instability for wavelengths below the ion Larmor scale. Phys. Rev. Lett. 114 (9), 2-6.

Landreman, M., Plunk, G.G. \& Dorland, W. 2015b Generalized universal instability: transient linear amplification and subcritical turbulence. J. Plasma Phys. 81 (5), 905810501, arXiv:1501. 02980 .

Landreman, M., Smith, H.M., Mollén, A. \& Helander, P. 2014 Comparison of particle trajectories and collision operators for collisional transport in nonaxisymmetric plasmas. Phys. Plasmas 21 (4), 042503.

Li, P.Y., Terry, P.W., Whelan, G.G. \& Pueschel, M.J. 2021 Saturation physics of threshold heat-flux reduction. Phys. Plasmas 28 (10), 102507.

LUXON, J.L. 2002 A design retrospective of the DIII-D tokamak. Nucl. Fusion 42 (5), 614-633.

McKinney, I.J., Pueschel, M.J., Faber, B.J., Hegna, C.C., TAlmadge, J.N., Anderson, D.T., MYNiCK, H.E. \& XANTHOPOULOS, P. 2019 A comparison of turbulent transport in a quasi-helical and a quasi-axisymmetric stellarator. J. Plasma Phys. 85 (5), 1-22. 
Nührenberg, J. 2010 Development of quasi-isodynamic stellarators. Plasma Phys. Control. Fusion 52 (12), 124003.

Nührenberg, J. \& ZILle, R. 1988 Quasi-helically symmetric toroidal stellarators. Phys. Lett. A 129 (2), 113-117.

Plunk, G.G., Connor, J.W. \& Helander, P. 2017 Collisionless microinstabilities in stellarators. Part 4. The ion-driven trapped-electron mode. J. Plasma Phys. 83 (4), 1-11, arXiv:1708.04085.

Plunk, G.G., Helander, P., Xanthopoulos, P. \& Connor, J.W. 2014 Collisionless microinstabilities in stellarators. III. The ion-temperature-gradient mode. Phys. Plasmas 21 (3), 032112, arXiv:1312.2424.

Proll, J.H.E., Helander, P., Connor, J.W. \& Plunk, G.G. 2012 Resilience of quasi-isodynamic stellarators against trapped-particle instabilities. Phys. Rev. Lett. 108 (24), 245002.

Proll, J.H.E., Xanthopoulos, P. \& Helander, P. 2013 Collisionless microinstabilities in stellarators. II. Numerical simulations. Phys. Plasmas 20 (12), 122506.

Pueschel, M.J., Li, P.Y. \& Terry, P.W. 2021 Predicting the critical gradient of ITG turbulence in fusion plasmas. Nucl. Fusion 61 (5), 054003.

Rosenbluth, M.N. 1968 Low-frequency limit of interchange instability. Phys. Fluids 11, 869.

TANG, W.M., CONNOR, J.W. \& HASTIE, R.J. 1980 Kinetic-ballooning-mode theory in general geometry. Nucl. Fusion 20 (11), 1439.

Terry, P.W., Faber, B.J., Hegna, C.C., Mirnov, V.V., Pueschel, M.J. \& Whelan, G.G. 2018 Saturation scalings of toroidal ion temperature gradient turbulence. Phys. Plasmas 25 (1), 012308.

Whelan, G.G., Pueschel, M.J. \& Terry, P.W. 2018 Nonlinear electromagnetic stabilization of plasma microturbulence. Phys. Rev. Lett. 120 (17), 175002.

Xanthopoulos, P., Bozhenkov, S.A., Beurskens, M.N.A., Smith, H.M., Plunk, G.G., Helander, P., Beidler, C.D., Alcusón, J.A., Alonso, J.A., Dinklage, A., et al. 2020 Turbulence mechanisms of enhanced performance stellarator plasmas. Phys. Rev. Lett. 125 (7), 075001.

Xanthopoulos, P., Cooper, W., Jenko, F., Turkin, Y., Runov, A. \& Geiger, J. 2009 A geometry interface for gyrokinetic microturbulence investigations in toroidal configurations. Phys. Plasmas $16(8), 082303$. 